\title{
Classification Analysis for Musical Instrument Signal
}

\author{
A.Muthumari \\ University College of Engineering, Anna University, Ramanathapuram - 623 513, India \\ muthu_ru@yahoo.com
}

\begin{abstract}
The automatic musical instrument classification taking place in a recording of music has many applications, together with music search through classes, music recommender methods and transcribers. Automatic instrument classification and identification of musical streams has become a difficulty research area over the last few years. In this approach is to classify the audio data based on the instruments. The audio features such as Enhanced Mel Frequency Cepstral Coefficient (EMFCC) and Enhanced Power Normalized Cepstral Coefficients(EPNCC) are used to extract the features for classification of various instrument classes. The classification algorithms such as J48, BFTree, $K$ Star, RandamForest and Bagging are used to classify musical instrument data into classes. Compare with various performance parameters like True Positive Rate, False Positive Rate etc., are used in various classification algorithms. The results shows that the best performance, almost $98 \%$ of accuracy, was attained by the classification system using the boosting technique with decision trees.
\end{abstract}

Key Word: Enhanced Mel Frequency Cepstral Coefficient, Enhanced Power Normalized Cepstral Coefficients, Support Vector Machines, Linear Predictive Coefficients.

\section{INTRODUCTION}

The automatic musical instrumental classification is an interesting and important sub problem in audio indexing and retrieval process. In the audio signal processing, the challenging task is the audio indexing and searching, which is usually combinations of overlying acoustic events, like music, speech, noise etc. the audio data is accessible at various sources like telephone conversations, newscasts, recordings of meetings, etc. and the amount of audio information on websites and other information storage is increasing daily. By using indexing and searching the digitized audio content users want to make the utmost of this ways. In the past years, utilising the computers it was challenging to recognize, interpretation, analyze digitized audio signal. The companies had to create and manually analyze written transcripts of audio data. However, with the evolution of higher storage volumes, faster processors and improved Speech signal processing methods it is likely to extract audio data using audio mining.

Audio instrument classification methods can have many potential purposes. For instance, detecting and inspecting solo passages can result in more knowledge about the distinct musical patterns and can be further utilized to deliver a basis for authors in music data. Many uses for editing of audio, retrieval audio/ video or transcription can be supported. A summary of audio understanding retrieval has been delivered by Foote [1] and extended by a few authors [2]. Other functions acoustic natural sound classification [3], and utilizing audio feature extraction to support video scene evaluation and annotation [4]. One of the vital primary aspects of instrument classification is to discover the correct feature extraction methods. For the period of last few years, the authors focused on recognition of speech in the audio signal, however few features can be applied to resolve the instrumentclassification problems.

In this paper, we present an investigation on instrument classification utilising machine learning algorithms. The extraction of features through using EMFCC - EPNCC method. Dataset of reduced features were then produced and their accuracy in instrumental classification was additional tested with a few classifiers utilising crossvalidations. Two feature schemes had been considered: Pitch and peak features. Our goal used to be to find the differences and synergies between the various feature schemes and experiment them with different classifiers, in order that a robust classification method could be built. Subsequent this introduction, in section 2 reviews the recent related work on musical instrument classification and feature analysis. In section 3 describe the approach of feature extraction methods used in this research work. Description of classification is in section 4. Experiment results and analysis based on the proposed methods are then presented in Section 5. Finally, in section 6 the findings, conclusions and future work of the proposed work.

\section{II.BACKGROUND}

The classification of twelve instrumental performed in various audios was studied in [2]. For classification, the author used multilayer neural networks and extraction of feature using the wavelet as well as MPEG-7 descriptors. This is used to be observed that a grouping of these double feature systems can drastically enhance the classification accurateness to a variety of range from $55 \%-98 \%$, by an average accurateness of $70 \%$. The misclassifications happened more commonly within every instrumentation. Brown et al. [6] carried out a study on identify 4 instruments are classified. 
The authors used for extraction of features using four methods namely autocorrelation and cepstral coefficients, spectral centroid and consistent $\mathrm{Q}$ transformation. Bayes decision tree algorithm was used for classification. The classification accuracy was ranges from $79 \%$ to $84 \%$. The authors [5] taught by classification instrument in isolated monophonic using k - NN classifier. The MFCC, Root-MeanSquare, consistent Q transform spectrum frequency, Multidimensional-Scaling, spectral centroid and amplitude envelope features are used. These features experienced essential component analysis for decrease to a complete dimensionality of 710 . The k-NN classifiers had been then learned beneath different ordered methods. A leave - one out approach used, acquiescent a correctness of $93 \%$ in instrumental classification. Agostini et al. [7] .The extraction of special features for classification of timbre and accuracy was measured by using Support Vector Machines, canonical discriminant analysis and k-NN with the primary and the last being the quality. In comparison with usual $55.7 \%$ accurate classification attained with certain conservatory scholars, the situation used to be argued that pc-based recognition of timbre can exceed human efficiency as a minimum for isolated tones.

The author presented that by a categorised classification methods, the recognition of categories such as grouping of instrumental was feasible. The method produced an order of nested clustering. The scheme began with the similar quantity of clustering as categories and then measured the distances among pairs of clustering. The neighbouring pairs had been then collected into new clustering. This progression was once persevered until all categories ay in a single clustering. The effort confirmed a correctness of 53\% for segmentation of music with based on the instruments played [8]. The above features are founded on extraction of coefficients that described quantity of the signal identification. Within the author there's an additional opinion of view for the extraction process. Relying on the building of the architectures, there are 2 main sets of often used features. The spectral characteristics utilized for frame based architectures [9], though the Linear Predictive Coefficients, Zero Crossing rate nd MFCCs utilized for the segment-based method architectures typically use the $[10,11]$. After the feature process that define the segmentations are offered to the classification process. There is a vast form of classification algorithm in the authors for audio classification in the instrument sounds. Some of the furthermost generally used classification methods are the Hidden Markov Model, SVMs, Gaussian Mixture Model, and Neural Networks approaches [12, 13, and 14].

\section{FEATURE EXTRACTION PROCESS}

The proposed audio signal classification strategy is evidently described in this part. Smoothening and filtering the input audio signal is carried out through mean filter. The extraction of pitch are carried out, in the view of the frequency difference of audio signal. At that point it's decided whether or not the pitch of the audio signal is fulfils the segment of signal, situated on the pitch frequency deviation. Using EMFCC-EPNCC the features are extracted for classification step with peak estimated signal of the feature. From this feature vector the classification classify the audio signal, based on the feature vector. From that classification output, the class of the musical instrument signal is identified. Fig. 1 illustrates the block diagram of proposed instrument classification method.

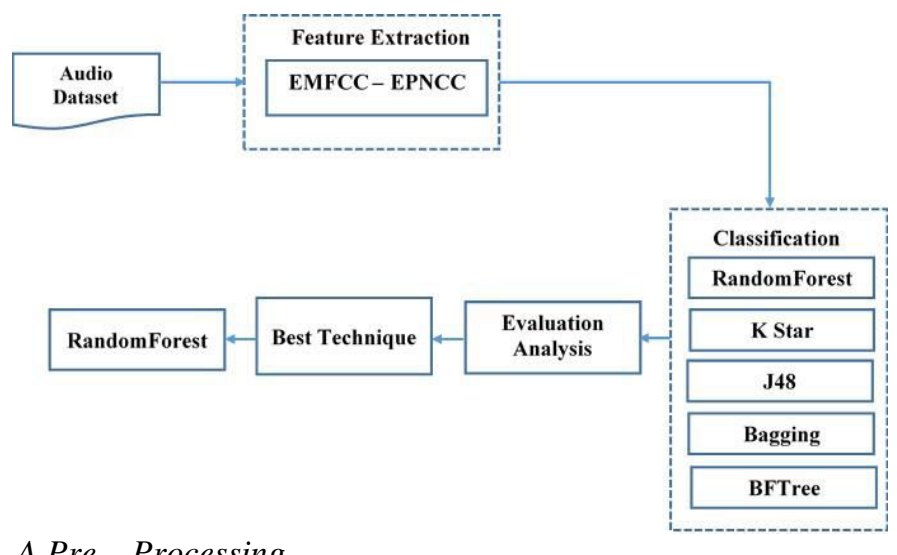

A.Pre-Processing

The audio signal is filtered using mean filter without knowing the statistical features of the signal. The audio signal is smoothened by seeing the mean values of the side windowing and changing the central windowing elements with the mean value. Then amplitude of signal is normalized and the gaussian noise existing in the signal is reduced. Gaussian is a very good assumption for every process or system that's subject to the Central Limit Theorem. First, because it does accurately reflect many systems. Second, because it is very easy to deal with mathematically, making it an attractive model to use. Gaussian noise is an excellent model of the time domain values of environmental background noise. In audio, the noise is most often colored, but the density is still well described by a Gaussian model. When you get into specific noises, like keyboard clicks, or engine noises, often there are non-Gaussian components, and so other models need to be used. But for high level general background noise, Gaussian is a great model.

R_Loc referred high peak positive

Q_Locreferred as negative edge of the audio signal

S_Locreferredas at the maximum signal difference at the negative point of input audio signal

Left \&Right as left and right positions of the sampled signal

Pitch extraction and peak extraction is done as proposed by [15]. Feature Vector is formed as

$\operatorname{Max}\left(\mathrm{Q}_{\text {loc }}\right)$, Max $\left(\mathrm{R}_{\text {loc }}\right)$, Max $\left(\mathrm{S}_{\text {loc }}\right)$,

Length $\left(\mathrm{Q}_{\text {loc }}\right)$, Length $\left(\mathrm{R}_{\mathrm{loc}}\right)$, Length $\left(\mathrm{S}_{\mathrm{loc}}\right)$,

Sum (Q_loc), Sum (R_loc), Sum (S_loc), whereas

$\mathrm{R}_{\text {loc }}=\operatorname{indx}_{\max }\left(\mathrm{X}_{1 \text { (Left } \rightarrow \text { Right })}\right)-1+$ Left

$\mathrm{Q}_{\text {loc }}=\operatorname{indx}_{\min }\left(\mathrm{X}_{1\left(\mathrm{Left} \rightarrow \mathrm{R}_{\text {loc }}\right)}\right)-1+$ Left (2)

$\mathrm{S}_{\text {loc }}=\operatorname{indx}_{\min }\left(\mathrm{X}_{1 \text { (Left } \rightarrow \text { Right })}\right)-1+$ Left (3)

here $X_{1}$-Input signal

Left $=\left\{\right.$ Pos $\left._{\text {Reg }}==1\right\}$

Right $=\left\{\right.$ Pos $\left._{\text {Reg }}==-1\right\}$

Pos $_{\text {Reg }}=\left\{X_{6}>\left(\right.\right.$ Thres $*$ Max $\left.\left._{h}\right)\right\}$

Thres $=\operatorname{Mean}\left(X_{6}\right)$

$\operatorname{Max}_{h}=\operatorname{Max}\left(X_{6}\right)$ 
$X_{1}=\frac{\left(\left(\sum_{m=0}^{N}\left(x_{1}(m) * h_{1}(m)\right)\right) *\left(T_{S}\right)\right)}{\left(\max \left(\left(\sum_{m=0}^{N}\left(x_{1}(m) * h_{1}(m)\right)\right) *\left(T_{S}\right)\right)\right)}$

Where $x_{1}$ the input is signal; $T_{S}$ is the sampling time of the signal; $N$ is number of samples in the signal; $h_{1}$ is low pass filter.

Within the extraction of pitch procedure, the objective function is applied to compute weight from the instrumental input signal situated on the cosine angle difference of the amplitude of the signal. The pre-allocated time samples are computed from the length of the input audio signal $\left(X_{i}\right)$ and pitch angle difference for every time sample, extracted founded on the objective function. At this point, modification in the drawback of time order by the Pitch $(t, \tau)$ computation and extracted pitch angle is found. The pitch signal is calculated from audio signal by using time domain based finding technique. Here Maximum Likelihood is used to extract the pitch features, and is denoted as,

$$
\operatorname{Pitch}(t, \tau)=\left\{\begin{array}{ll}
\frac{1}{N+1} \sum_{n=0}^{N} \operatorname{Pitch}(t+n \tau) & 0 \leq t \leq b \\
\frac{1}{N} \sum_{n=0}^{N} \operatorname{Pitch}(t+n \tau) & b \leq t \leq \tau
\end{array}\right\}
$$

Here, " $t$ " is sampling time in the instrumental signal, " $\tau$ " is the size of the frame in the input signal; " $N$ " is total size of the instrumental input signal; " $b$ " is one segment of length.

\section{A. Feature Extraction method}

This enhanced feature extraction method is used to process from the audio samples at fs (HZ) to extract feature. An audio signal is divided into frames using windowing process and analysis of spectrum is done for each and every frame of the signal. The Discrete Fourier Transform (DFT) isuseful on the frame. The Mel frequency warping output is got from the DFT output.

The transform output are extracted from the Mel Cepstrum, within the EMFCC based on feature extraction process. The output of the signal is split into frames by windowing process at fixed intervals. The distribution function characterized by window is given as

$P_{i}(k)=\left(\frac{1}{N}\right)\left|X_{i}(k)\right|^{2}$

Windowing minimizes the disturbances at beginning and last point of the frame. The window function output is given as

$Y(n)=X(n) W(n)$

Where $0 \leq \mathrm{n} \leq \mathrm{N}-1$. Now, ' $N$ ' indicates the number of samples, $Y(n)$ denotes the output signal. The input of the signal is $X(n)$ and the windowing is $W(n)$.

Coefficients are computed by using Fast Fourier Transform (FFT) from the log Mel cepstrum. Finding the Mel frequency warping form is given as

$\operatorname{mel}(f)=2595 \cdot \log \left(1+\frac{\omega f_{s}}{2 \pi \cdot 700 \mathrm{~Hz}}\right)$

Where ' $f_{s}$ ' denotes the frequency of the sample and ' $\omega$ ', denoted the warping function. To be combined the cosine transformation function, the Mel frequency warping function is normalized to fulfil the particular measure $\tilde{\mu}(\pi)=\pi$.

$$
\begin{aligned}
& \tilde{\mu}(\omega)=\frac{\pi}{\mu(\pi)} \cdot \mu(\omega) \\
& =d \cdot \log \left(1+\frac{\omega \mathrm{f}_{\mathrm{S}}}{2 \pi \cdot 700 \mathrm{~Hz}}\right)
\end{aligned}
$$

Where $d=\frac{\pi}{\log \left(1+\frac{f s}{2 * 700 H z}\right)}$

At that point, Mel Cepstrum output of audio signal is extracted using the inverse DFT from EMFCC function. The audio signal feature yield was calculated from the product from the cosine transformation, logarithmic of the magnitude and filter coefficient.

$M_{F}=C T * \log (M a g) * \operatorname{diagonal}(F L)(11)$

Where $\mathrm{M}_{F}$ the audio feature is output; CT is the cosine transform; $M a g$ is the magnitude of FFT; FL is the filter coefficients.

\section{CLASSIFICATION ALGORITHMS}

\section{A. Randomforest}

Random forests are a mixture of tree predictors such every tree depends on the values of a random vector sampled independently and with constant distribution for all trees within the forest. The generalization error for forests converges to a limit as the variety of trees within the forest becomes large.

The key idea of the regularization framework is to penalize choosing a new feature for splitting once its gain is similar to the features utilized in previous splits. The regularization structure is useful on random forest and boosted trees here, and may be simply applied to different tree models. Experimental studies show that the regularised trees can choose high-quality feature subsets with relevance each robust and weak classifiers. Because tree models can naturally handle categorical and numerical variables, lost values, totally different scales between variables, interactions and nonlinearities the tree regularization structure provides an effective and efficient feature choice solution for several practical issues [16] [17] [18] [19].

\section{A. J48 Decision tree}

J48 is an extension lead of ID3. The further features of J48 are accounting for missing values, derivation of rules, continuous attribute value ranges, decision trees pruning and so forth. Within the WEKA data mining tool, J48 is an open source Java implementation of the C4.5 algorithm. The WEKA tool delivers a number of choices related to tree pruning. In case of possible over fitting pruning can be used as a tool for summarizing. In other algorithms the classification is done recursively till each single leaf is pure, that's the classification of the information should be as best as viable. This method it produces the rules from which precise identification of that data is produced. The objective is regularly generalization of a decision tree unless it positive aspects equilibrium of flexibility and accuracy.

\section{B.The $K^{*}$ Algorithm}

The $K^{*}$ algorithm is defined as a process of clustering process which main aims at the split of ' $n$ ' observation into ' $k$ ' clusters in which every observation belongs to the cluster with the nearest mean. We are able to describe $K^{*}$ algorithm as an example based trainer which makes use of entropy as a distance measure. The advantages are that it presents a regular technique to dealing with of missing values, symbolic attributes and actual valued attributes [20]. 


\section{EXPERIMENTAL RESULTS}

\section{A. Database}

The musical dataset used for evaluation of proposed approach consist of different instrumental signal collected from ffuhrmann [21]. The dataset contains 1100 audio files of instrumental signal with 11 classes. These audio file classes are cel (100), cla (100), flu (100), gac (100), gel (100), org (100), pia (100), sax (100), tru (100), vio (100), and voi (1000). For training $80 \%$ of audio files are selected from the dataset and the remaining $20 \%$ audio files are used as the testing set. The experimental platform is a Laptop with Intel Core 2 Duo, $2.20 \mathrm{GHz}, 2.00 \mathrm{~GB}$ RAM, 32 Bit operating system and Windows 7 Ultimate. The proposed work was implemented in the MATLAB environment. The properties of audio signal used in the proposed work is shown in Table I.

TABLE I. A SUMMARY OF AUDIO SIGNAL PROPERTIES
\begin{tabular}{|l|c|}
\hline Audio Property & Value (ffuhrmann) \\
\hline Audio Bit Rate & $705 \mathrm{kbps}$ \\
\hline Audio Format & .wav \\
\hline Audio Length & $3 \mathrm{sec}$ \\
\hline Audio sample Size & $16 \mathrm{bit}$ \\
\hline Channel & Mono \\
\hline
\end{tabular}

\section{B. Performance Evaluation}

In this, the classification of pre-processing is applied based on all values of taken 41 features. A comparative study of classification accuracy in J48, BFTree, K Star, RandamForest and Bagging algorithm is applied in this work. The accuracy measures like TP rate, FP rate, precision, recall F- measure and ROC Area evaluation is also applied. The several formula used for the calculation of different measures are as follows.

$$
\begin{gathered}
\text { TP }=\frac{d}{c+d} \\
\text { TP }=\text { True Positive } \\
\text { FP }=\frac{b}{a+b} \\
\text { FP }=\text { False Positive } \\
\text { TN }=\frac{a}{a+b} \\
\text { TN }=\text { True Negative } \\
\text { FN }=\frac{c}{c+d} \\
\text { FN }=\text { False Negative }
\end{gathered}
$$

a) Precision:

$$
\text { Precision }=\frac{\mathrm{TP}}{(\mathrm{TP}+\mathrm{FP})}
$$

b) Recall:

$$
\text { Recall }=\frac{\mathrm{TP}}{(\mathrm{TP}+\mathrm{FN})}
$$

c) Sensitivity:

$$
\text { Sensitivity }(\%)=\frac{\mathrm{TP}}{\mathrm{TP}+\mathrm{FN}} \times 100
$$

d) Specificity:

$$
\text { Specificity }(\%)=\frac{\mathrm{TN}}{\mathrm{TN}+\mathrm{FP}} \times 100
$$

e) Accuracy:

$$
\text { Accuracy }(\%)=\frac{\text { Number of correctly classified results }}{\times 100}
$$

f) F-Score:

$$
\text { F1 Score }=\frac{2 *(\text { Recall } * \text { Precision })}{\text { Recall }+ \text { Precision }}
$$

The musical instrument classification results are discussed in this part. A confusion matrix is a unique table design that includes information regarding actual and estimated classifications carried out by means of audio instrumentclassification system. Table II indicates the confusion matrix of RandamForest method. Within the confusion matrix, every column represents theinstances in percentage a predicted class, whereasevery row represents the instances in percentage anactual classification.

TABLE II

CONFUSION MATRIX OF RANDAMFOREST ALGORITHM

\begin{tabular}{|c|c|c|c|c|c|c|c|c|c|c|c|}
\hline \multirow{2}{*}{ Actual } & \multicolumn{9}{|c|}{ Classification } \\
\cline { 2 - 14 } & Cel & Cla & Flu & Gac & Gel & Org & Pia & Sax & Tru & Vio & Voi \\
\hline Predicted & $\mathbf{9 9}$ & 1 & 0 & 0 & 0 & 1 & 4 & 0 & 1 & 0 & 0 \\
\hline Cel & 0 & $\mathbf{9 8}$ & 0 & 0 & 0 & 0 & 0 & 0 & 0 & 0 & 0 \\
\hline Fla & 1 & 0 & $\mathbf{1 0 0}$ & 2 & 0 & 0 & 0 & 0 & 0 & 0 & 0 \\
\hline Gac & 0 & 0 & 0 & $\mathbf{9 8}$ & 1 & 0 & 0 & 0 & 0 & 2 & 1 \\
\hline Gel & 0 & 0 & 0 & 0 & $\mathbf{9 6}$ & 1 & 0 & 0 & 0 & 0 & 0 \\
\hline Org & 0 & 0 & 0 & 0 & 1 & $\mathbf{9 8}$ & 1 & 0 & 0 & 0 & 0 \\
\hline Pia & 0 & 1 & 0 & 0 & 0 & 0 & $\mathbf{9 5}$ & 0 & 0 & 0 & 0 \\
\hline Sax & 0 & 0 & 0 & 0 & 0 & 0 & 0 & $\mathbf{1 0 0}$ & 0 & 0 & 0 \\
\hline Tru & 0 & 0 & 0 & 0 & 0 & 0 & 0 & 0 & $\mathbf{9 9}$ & 0 & 0 \\
\hline Vio & 0 & 0 & 0 & 0 & 0 & 0 & 0 & 0 & 0 & 97 & 0 \\
\hline Voi & 0 & 0 & 0 & 0 & 2 & 0 & 0 & 0 & 0 & 1 & $\mathbf{9 9}$ \\
\hline
\end{tabular}

To classify the instruments correctly from the training dataset, accuracy and error rates are estimated using classification methods. The accuracy of Random Forest $98 \%$, K Star $95 \%$, J48 75\%, Bagging 72\% and BFTree 62\%. The confusion matrix used to find the several estimation measures such as TP, FP, accuracy, recall, precision, F-Measure and ROC Area. Table III shows the TP and FP rate of Random Forest algorithm. 
TABLE III

TP AND FP RATE OF RANDAMFOREST ALGORITHM

\begin{tabular}{|c|c|c|c|c|c|c|}
\hline Class & TP Rate & FP Rate & Precision & Recall & F-Measure & ROC Area \\
\hline Cel & 0.99 & 0.007 & 0.934 & 0.99 & 0.961 & 0.999 \\
\hline Cla & 0.98 & 0 & 1 & 0.98 & 0.99 & 0.999 \\
\hline Flu & 1 & 0.003 & 0.971 & 1 & 0.985 & 1 \\
\hline Gac & 0.98 & 0.004 & 0.961 & 0.98 & 0.97 & 1 \\
\hline Gel & 0.96 & 0.001 & 0.99 & 0.96 & 0.975 & 1 \\
\hline Org & 0.98 & 0.002 & 0.98 & 0.98 & 0.98 & 1 \\
\hline Pia & 0.95 & 0.001 & 0.99 & 0.95 & 0.969 & 0.998 \\
\hline Sax & 1 & 0 & 1 & 1 & 1 & 1 \\
\hline Tru & 0.99 & 0 & 1 & 0.99 & 0.995 & 1 \\
\hline Vio & 0.97 & 0 & 1 & 0.97 & 0.985 & 1 \\
\hline Voi & 0.99 & 0.003 & 0.971 & 0.99 & 0.98 & 1 \\
\hline Weighted Avg. & 0.981 & 0.002 & 0.981 & 0.981 & 0.981 & 1 \\
\hline
\end{tabular}

Table IV depicts the correct and wrong prediction of classification algorithms. Table $\mathrm{V}$ illustrations of the weighted average accuracy of the classification methodsfor the musical instruments. The classification performance for every of the measured classification methods is presented in Table.

TABLE IV

CORReCt ANd Wrong PREDiction Of Classification Algorithm

\begin{tabular}{|c|c|c|c|c|c|c|c|c|c|c|}
\hline \multirow{2}{*}{$\underset{\text { Classes }}{\stackrel{\text { Classifier }}{\longrightarrow}}$} & \multicolumn{2}{|c|}{ BFTree } & \multicolumn{2}{|c|}{ Bagging } & \multicolumn{2}{|c|}{ J48 } & \multicolumn{2}{|c|}{ K Star } & \multicolumn{2}{|c|}{ RaudamForest } \\
\hline & Correct & Wrong & Correct & Wrong & Correct & Wrong & Correct & Wrong & Correct & Wroug \\
\hline Cel & 82 & 18 & 77 & 23 & 81 & 19 & 100 & 0 & 99 & 1 \\
\hline $\mathrm{Cla}$ & 70 & 30 & 68 & 32 & 84 & 16 & 95 & 5 & 98 & 2 \\
\hline Flu & 65 & 35 & 70 & 30 & 81 & 19 & 93 & 7 & 100 & 0 \\
\hline Gac & 80 & 20 & 75 & 25 & 78 & 22 & 98 & 2 & 98 & 2 \\
\hline Gel & 54 & 46 & 75 & 25 & 82 & 18 & 93 & 7 & 96 & 4 \\
\hline Org & 62 & 38 & 83 & 17 & 83 & 17 & 96 & 4 & 98 & 2 \\
\hline Pla & 62 & 38 & 61 & 39 & 61 & 39 & 91 & 9 & 95 & 5 \\
\hline $\operatorname{Sax}$ & 42 & 58 & 63 & 37 & 63 & 37 & 92 & 8 & 100 & 0 \\
\hline Iтu & 47 & 57 & 60 & 40 & 74 & 26 & 94 & 6 & 99 & 1 \\
\hline Vio & 54 & 46 & 75 & 25 & 69 & 29 & 97 & 3 & 97 & 3 \\
\hline Voi & 60 & 40 & 83 & 17 & 73 & 27 & 91 & 9 & 99 & 1 \\
\hline Percentage & 61.64 & 38.73 & 71.82 & 28.18 & 75.36 & 24.45 & 94.55 & 5.45 & 98.09 & 1.91 \\
\hline
\end{tabular}

TABLE V

ACCURACY By WeIGHTED AVERAGE OF ClassifIERS

\begin{tabular}{|c|c|c|c|c|c|c|}
\hline Class & TP Rate & FP Rate & Precision & Recall & F- Measure & ROC Area \\
\hline BFTree & 0.616 & 0.038 & 0.67 & 0.616 & 0.622 & 0.945 \\
\hline Bagging & 0.718 & 0.028 & 0.729 & 0.718 & 0.718 & 0.969 \\
\hline J48 & 0.754 & 0.025 & 0.759 & 0.754 & 0.752 & 0.983 \\
\hline K Star & 0.945 & 0.005 & 0.966 & 0.945 & 0.951 & 0.998 \\
\hline RandamForest & 0.981 & 0.002 & 0.981 & 0.981 & 0.981 & 1 \\
\hline
\end{tabular}

TABLE VI

Musical Instrument Classification Performance (Percentage)

\begin{tabular}{|c|c|c|c|c|c|}
\hline Classifier & RandamForest & K Star & J48 & Bagging & BFTree \\
\hline Performance & 98.09 & 94.55 & 75.36 & 71.82 & 61.64 \\
\hline
\end{tabular}

\section{A. References}

The scopes of few present work and performance attained are shown in Table VII, here the number of instruments and classification performance of musical instrument classifications are record out. It may be noticeable that our outcomes are higher than or similar with those achieved by other researchers. Though it can noted that the number of instruments included is differentand that the datasets are different althoughthat these kind of included fuhrmann dataset.

TABLE VII

Performance (Percentage) Of Instrument Classification Compared

\begin{tabular}{|l|c|c|}
\hline \multicolumn{1}{|c|}{ Work } & $\begin{array}{c}\text { No. of } \\
\text { Instruments }\end{array}$ & $\begin{array}{c}\text { Performanc } \\
\text { e }\end{array}$ \\
\hline Eronen [6] & 29 & 77 \\
\hline Martin and Kim [23] & 14 & 90 \\
\hline Agostini et al. [7] & 27 & 81 \\
\hline Kostek [2] & 12 & 70 \\
\hline Kaminskyj and Czaszejko [5] & 19 & 97 \\
\hline Benetos et al. [18] & 6 & 95.2 \\
\hline Deng et al. [22] & 20 & 96.5 \\
\hline This work & 11 & 98.09 \\
\hline
\end{tabular}

\section{CONCLUSION}

This work measure the performances in terms of classification accuracy of J48, BFTree, K Star, RandamForest and Bagging algorithms using several accuracy measures like TP rate, FP rate, precision, recall F- measure and ROC Area. In the implementation system, it is viewed best numerical values of some attributes within the musical instrument data. The experiment result indicates that the accuracy $98 \%$ is observed in RandomForest, accuracy $95 \%$ is found in K Star, accuracy $75 \%$ isfound in J48, accuracy $72 \%$ is found Bagging and accuracy $62 \%$ is found in BFTree classifier algorithm.

Based on the classification results of the entire five algorithms, the efficiency of RandamForest is better than the other four algorithms for the chosen data set. In future we will use some more datasets to validate the proposed method. Only 1100 data have been used in this proposed work. In future work a huge number of dataset too reflected.

\section{REFERENCES}

[1] J. Foote, "An overview of audio information retrieval," Multimedia Syst., vol. 7, no. 1, pp. 2-10, Jan. 1999.

[2] B. Kostek, "Musical instrument classification and duet analysis employing music information retrieval techniques," Proc. IEEE, vol. 92, no. 4,pp. 712-729, Apr. 2004.

[3] L. Ma, B. Milner, and D. Smith, "Acoustic environment classification," ACM Trans. Speech Lang. Process., vol. 3, no. 2, pp. 1-22, Jul. 2006.

[4] A. Divakaran, R. Regunathan, Z. Xiong, and M. Casey, "Procedure for audio-assisted browsing of news video using generalized sound recognition," Proc. SPIE, vol. 5021, pp. 160-166, 2003.

[5] I. Kaminskyj and T. Czaszejko, "Automatic recognition of isolated monophonic musical instrument sounds using kNNC," J. Intell. Inf. Syst., vol. 24, no. 2/3, pp. 199-221, Mar. 2005.

[6] J. C. Brown, O. Houix, and S. McAdams, "Feature dependence in the automatic identification of musical woodwind instruments," J. Acoust. Soc. Amer., vol. 109, no. 3, pp. 1064-1072, Mar. 2001. 
[7] G. Agostini, M. Longari, and E. Poolastri, "Musical instrument timbres classification with spectral features," EURASIP J. Appl. Signal Process, vol. 2003, no. 1, pp. 5-14, 2003.

[8] S. Essid, G. Richard, and B. David. Instrument recognition in polyphonic music based on automatic taxonomies. In IEEE Transactions on Audio, Speech, and Language Processing, volume 14, pages 68-80, January 2006.

[9] E. Dogan, M. Sert and A. Yazici, "Content-Based Classification and Segmentation of Mixed-Type Audio by Using MPEG-7 Features", In Proceedings of the 2009 First International Conference on Advances in Multimedia, pp. 152-157, 2009.

[10] J. Huang, Y. Dong, J. Liu, C. Dong and H. Wang, "Sports audio segmentation and classification", In Proceedings of the IEEE International Conference on Network Infrastructure and Digital Content, IC-NIDC 2009, pp. 379-383, 2009.

[11] R. Huang and J.H.L. Hansen, "Advances in unsupervised audio segmentation for the broadcast news and NGSW corpora", In Proceedings of the IEEE International Conference on Acoustics, Speech, and Signal Processing, ICASSP 2004, vol. 1, 2004.

[12] H. Meinedo and J. Neto, "Audio Segmentation, Classification and Clustering in a Broadcast News Task", In Proceedings of the 2003 IEEE International Conference on Acoustics, Speech, and Signal Processing, ICASSP 2003, pp. 5-8, 2003.

M. Liu, C. Wan and L. Wang, "Content-based audio classification and retrieval using a fuzzy logic system: towards multimedia search engines", Soft Comput., vol. 6, no. 5, pp. 357-364, 2003.

[13] H. Aronowitz, "Segmental modeling for audio segmentation", In Proceedings of the IEEE International Conference on Acoustics, Speech and Signal Processing, ICASSP 2007, pp. 393 -396, 2007.

[14] Muthumari, A., Mala, K.: 'An Efficient Approach for Segmentation, Feature Extraction and Classification of Audio Signals', Circuits and Systems, 2016, Vol. 7, pp. 255-279.
[15] Tarannum A Bloch, Prof.V.B.Vaghela, Dr.K.H.Wandra, "Applied Taxonomy Techniques Intended for Strenuous Random Forest Robustness", Int. J. Comp. Tech. Appl., Vol 2 (6), pp-2061-2065, 1998.

[16] SnehLataPundir, Amrita "Feature Selection Using Random Forest in Intrusion Detection System" International Journal of Advances in Engineering \& Technology, Vol. 6, Issue 3, pp. 1319-1324, July 2013.

[17] AnubhaDubey, Bhaskar Pant, UshaChouhan, "Machine learning model for HIV1 and HIV2 enzyme secondary structure classification", J. Comput. Method. Mol. Design, vol. 1 (2): pp- 1-8, 2011.

[18] Mr.HirenGadhvi, Ms.Madhu Shukla "Comparative Study of Classification Algorithms for Web Spam Detection", International Journal of Engineering Research \& Technology (IJERT), Vol. 2 Issue 12, pp- 2497-2501, December - 2013.

[19] Trilok Chand Sharma, Manoj Jain, "WEKA Approach for Comparative Study of Classification Algorithm".

[20] 'ffuhrmann',http://www.dtic.upf.edu/ ffuhrmann/PhD/data/, Accessed 21-08-2015.

[21] D. Deng, Simmermacher, And Cranefield A Study On Feature Analysis For Musical Instrument Classification IEE Transactions On Systems, Man, And Cybernetics: Cybernetics, Vol. 38, No. 2, pp. 429-438, April 2008.

K. D. Martin, Y. E. Kim, "Musical instrument identification: A patternrecognition approach", in $136^{\text {th }}$ meeting of the Acoustical Society of America, Norfolk, Virginia, USA, 1998. 